\title{
5. AO-Seminar West in Köln am 30.01.2019
}

Lars-Peter Müller, Gereon Schiffer, Joachim Windolf

Zum 5. Mal fand am 30.01.2019 das AO-Seminar West unter der Leitung von Gereon Schiffer, Lars Müller und Joachim Windolf statt. Als anatomische Region stand in diesem Jahr die Klavikula mit angrenzenden Gelenken auf dem Programm. Auch didaktisch gab es etwas Neues: Die Referenten wurden im Vorfeld aufgefordert, ihre Präsentationen vollständig über konkrete Fällen aufzubauen. Um es vorwegzunehmen: Das Konzept ging auf. In Kombination mit dem häufig eingesetzten und von Henry Langer gewohnt perfekt bedienten ARS-Abstimmsystem und dem traditionell sehr diskussionsfreudigen Kölner Publikum entwickelte sich über 5 Stunden ein lebhafter, kontroverser, stets kollegialer und unterhaltsamer Dialog zwischen Referenten, Chairmen und den fast 60 Teilnehmern.

Den Einstieg lieferte Jonas Andermahr (Mechernich), der als einziger vom fallbasierten Konzept ausgenommen war, weil er die Anatomie der Klavikula als Thema hatte. Aus seiner langjährigen Beschäftigung mit der Materie an der Kölner Anatomie konnte er den Zuhörer viele neue anatomische Details vermitteln. Oder wussten Sie, dass die Klavikula der Knochen ist, der in der Skelettentwicklung einerseits als erster ossifiziert und andererseits als letzter seine Wachstumsfugen schließt?

Der 1. Block beschäftigte sich dann mit den Luxationen des Sternoklavikulargelenks. Torsten Gerich, in Luxemburg tätiger Schulterchirurg, verwies auf die insgesamt sehr geringen Fallzahlen bei dorsaler Luxation, aber auch auf die Möglichkeit schwerwiegender, insbesondere vaskulärer Komplikationen bei der Therapie. Der Herz-Thorax-Chirurg sollte immer informiert sein bei derartigen Eingriffen. Gerich empfahl für die dorsale Luxation die Verwendung einer lateralen Klavikulaplatte, die nur an der Klavikula, nicht jedoch über dem Manubrium mit Schrauben fixiert wird, da die beidseitige Fixation nach seinen Erfahrungen regelhaft zu einer Lockerung mit ossärer Destruktion und damit zu einem Versagen der Therapie führt. In seiner kleinen Fallstudie konnte er von bislang ermutigenden Ergebnissen berichten.

Peter Schmittenbecher (Karlsruhe) stellte anschließend eine Fülle von kindlichen Klavikulafrakturen vor. Hier gilt es, nicht nur die Wünsche der jungen Patienten, sondern auch die der Eltern zu berücksichtigen. Bei den Kindern funktioniert weiterhin sehr viel konservativ, auch eher

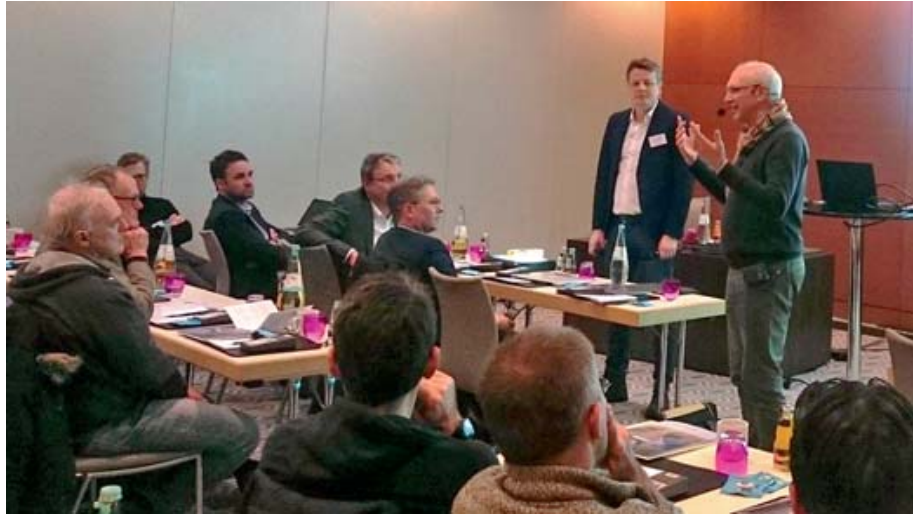

- Abb. 1 Diskussion mit Peter Schmittenbecher. Quelle: Henry Langer, AO Foundation

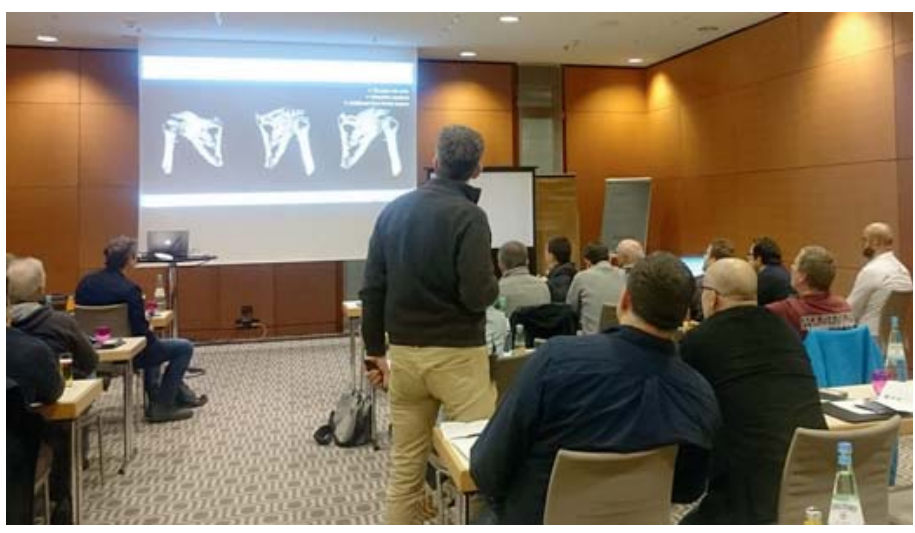

- Abb. 2 Falldiskussion mit Martin Jaeger. Quelle: Henry Langer, AO Foundation

ungewöhnliche Techniken wie die PDS-Cerclage bei Schaftfrakturen führen hier zu sehr guten Resultaten. Schmittenbecher empfahl, bei der Indikationsstellung und Methodenauswahl hier neben dem Lebensalter auch den Entwicklungsstand in der Pubertät hinzuzuziehen, um zu entscheiden, ob der junge Mensch noch unter die kindlichen Strategien und Techniken fällt oder nach den Prinzipien der Erwachsenenchirurgie zu behandeln ist.

Im 2. Block präsentierten Axel Jubel (Köln), Michael Hackl (Köln) und Ulrich Thelen (Bergisch Gladbach) Fälle und 


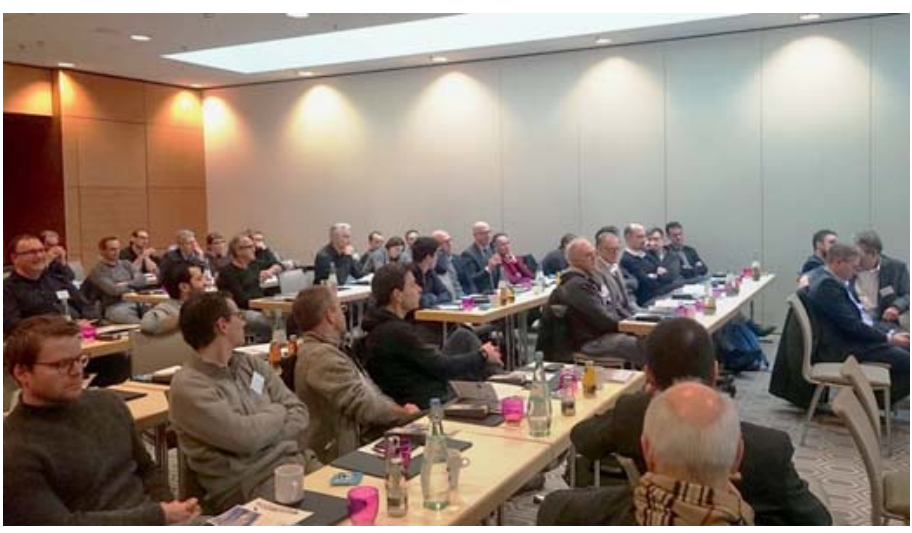

Abb. 3 Diskussion im Sitzungssaal. Quelle: Henry Langer, AO Foundation

Verläufe von Frakturen des Klavikulaschaftes. Übereinstimmend mit dem Auditorium stellte man fest, dass aktuell deutlich mehr am Klavikulaschaft operiert wird als noch vor Jahren. Dies hängt auch und gerade mit der veränderten und belastbareren Studienlage zusammen. Jubel wies auf das in randomisierten Studien nachgewiesene deutlich höhere Pseudarthrosenrisiko hin, welches mit zunehmendem Alter und Komplexität der Fraktur deutlich im 2-stelligen Prozentbereich anzusiedeln ist. Auch der Anspruch des Patienten bezüglich schneller Belastbarkeit spielt eine Rolle. Längeninstabile Frakturen sind weiterhin sehr gut mit winkelstabilen Platten versorgt, für die einfache dislozierte Fraktur empfahlen die Referenten die ESIN-Technik. Verkürzungen von $2 \mathrm{~cm}$ und mehr sollten vermieden werden.

Die laterale Klavikula einschließlich Akromioklavikulargelenk und dem ligamentären Aufhängeapparat (SSSC = Superior Shoulder Suspensory Complex) war Gegenstand der letzten Session nach der Fingerfood-Pause, den Martin Jaeger (Freiburg) mit interessanten und komplexen Fällen und einem sehr engagierten Vortrag einleitete. Seine Herangehensweise an diese komplexen Kombinationsverletzungen mit einer subtilen Analyse des Unfallmechanismus („steht die Klavikula hoch oder der Rest der Schulter tief?") überzeugten das Publikum, auch wenn sich Jaeger auf kein einfach zu reproduzierendes Rezept fest- nageln lassen wollte. Hier ist offensichtlich noch auf längere Zeit „Individualmedizin“, extensive Diagnostik und genaue Nachbeobachtung des Patienten angesagt.

Philipp Kobbe (Aachen) warf zuletzt noch ein kritisches Schlaglicht auf die aktuell diskutierten Therapien der ohnehin problematischen Verletzungen des AC-Gelenks. Nach wie vor gibt es keinen klaren Konsens hinsichtlich der OP-Indikation bei den Rockwood-3/4-Verletzungen einerseits und der OP-Strategie bzw. den zu verwendenden Implantaten bei den Rockwood-5-Läsionen andererseits. Die zuletzt stark in Mode gekommenen und von der Industrie offensiv beworbenen Fadenanker-Methoden weisen nach Übereinstimmung im Plenum eine recht flache Lernkurve und nicht unerhebliche Komplikationsmöglichkeiten auf. Dies konnte Kobbe mit mehreren Fallbeispielen von Fehlschlägen eindrucksvoll demonstrieren und mit aktueller Literatur untermauern. Wahrscheinlich werden zu den über 150 bekannten Operationsmethoden für diese Verletzungen noch weitere hinzukommen.

Teilnehmer und Chairmen äußerten sich mit dem Verlauf der „wochenendsparenden Fortbildung“ äußerst zufrieden, das neue, komplett fallbasierte Konzept wurde sehr positiv angenommen. Trotz der großen Teilnehmerzahl konnte ein „Seminar“-Charakter erzielt werden, da es zu allen Themen eine Vielzahl von willigen Diskutanten gab. Möglich wurde dies durch die Bereitschaft der Referenten, sich auf dieses neue Format einzulassen. Besonders positiv und fast einmalig aus Sicht der Chairmen: Kein einziges Mal wurde der Fall aus einem „auswärtigen Krankenhaus“ vorgestellt, alle Redner zeigten Fälle aus den eigenen Kliniken!

Prof. Dr. Lars-Peter Müller, Köln

Dr. Gereon Schiffer, Bergisch-Gladbach

Prof. Dr. Joachim Windolf, Düsseldorf

Bibliografie

DOI https://doi.org/10.1055/a-0874-3407

OP-JOURNAL 2019; 35: 75-76 @ Georg Thieme Verlag KG Stuttgart · New York ISSN 0178-1715 